\title{
Quantification of the deuterium ion fluxes from a plasma source
}

\author{
A Manhard, T Schwarz-Selinger and W Jacob \\ Max-Planck-Institut für Plasmaphysik, EURATOM Association, Boltzmannstr. 2, 85748 \\ Garching, Germany \\ E-mail: armin.manhard@ipp.mpg.de
}

\begin{abstract}
We present an electron-cyclotron-resonance plasma source with a biased sample holder that is well suited for the implantation of deuterium ions into tungsten. Achievable energies range from several $\mathrm{eV}$ up to $200 \mathrm{eV}$ per deuteron. The deuterium ion fluxes from this plasma source were thoroughly quantified with a retarding field analyser and a plasma monitor, i.e. an energy resolving mass spectrometer. We present the results of these measurements for different microwave input powers, $\mathrm{D}_{2}$ gas pressures and sample bias settings. Typical achievable deuteron fluxes are of the order of $10^{19}$ to $10^{20} \mathrm{D} \mathrm{m}^{-2} \mathrm{~s}^{-1}$ and are predominantly carried by $\mathrm{D}_{3}^{+}$ions. Additionally, an attempt to quantify the flux of neutral deuterium atoms to the sample surface is presented.
\end{abstract}

PACS numbers:

52.50.Dg Plasma sources

52.35.Hr Electromagnetic waves (e.g., electron-cyclotron, Whistler, Bernstein, upper hybrid, lower hybrid)

52.70.Nc Particle measurements

52.35.Qz Microinstabilities (ion-acoustic, two-stream, loss-cone, beam-plasma, drift, ion- or electron-cyclotron, etc.)

Submitted to: Plasma Sources Sci. Technol.

\section{Introduction}

Plasma discharges are widely used in technical processes such as surface modification and thin-film deposition and etching. The quantitative characterisation of plasma sources is a topic of particular importance for a fundamental understanding of plasma sources as well as for a technological optimisation of processes. Hydrogen plasmas play a certain role in technical discharges so that a precise determination of the absolute hydrogen fluxes to a plasma-treated surface is very desireable. This is important for understanding both the discharge mechanism and the plasma-induced processes at the surface. Hydrogen plasmas are frequently used for erosion of carbon and carboncontaining films. In particular in fusion research, erosion of carbon surfaces due to interaction with the hydrogen isotopes from the fusion plasma is an important plasmasurface-interaction (PSI) process [1]. In recent years, interest in fusion PSI research has 
shifted to the interaction of hydrogen isotopes with tungsten materials because tungsten is a promising candidate as a first wall armor material in fusion devices. An issue of significant importance is to investigate the retention of hydrogen isotopes in tungsten. Especially the radioactive tritium which will be used as fuel for the fusion reaction is a potential hazard [2]. Retention is often studied by implanting tungsten samples with a deuterium ion beam. This allows to select a single ion species with a well defined kinetic energy $[3,4]$. Ion beams usually have a distinct beam profile and a rather limited size. Furthermore, the ion flux is inhomogeneous near the edges of the implantation spot as shown by [5]. This leads to some difficulties for the subsequent analysis of the deuterium inventory. For example, in nuclear reaction analysis, which is in most cases used to determine the implanted deuterium depth profile, it is not trivial to assure that the measurement spot hits the center of the implantation spot. In thermal desportion spectroscopy (TDS), which is often used to measure the total deuterium inventory, the signal is determined by the total implanted amount and, therefore, proportional to the irradiated sample surface. Because TDS often suffers from low signal-to-noise ratios it would be much more convenient to have larger samples with laterally homogeneous implantation. Another challenge with ion beam implantation setups is that at low ion energies the ion flux becomes very low due to space charge limitation leading to unacceptably long exposure times to accumulate relevant deuterium fluences of up to the order of $10^{25} \mathrm{D} \mathrm{m}^{-2}$ on the sample.

High fluxes at low ion energies can be achieved by exposing the samples to a lowtemperature plasma. A much larger area can be exposed in order to achieve larger TDS signals. If sufficient lateral homogeneity of the plasma is given, even multiple samples can be implanted simultaneously. This allows comparing the retention behaviour of different sample types under exactly the same conditions. Furthermore, the energies of ions extracted from a plasma can be easily reduced to the sheath potential without a strong reduction in flux. Energies as low as a few eV can easily be achieved in this way. Such low energies at high fluxes are not possible with ion beam set-ups, but are prevalent in the so-called detached divertor operation regime of present day and future fusion experiments [2]. Co-implantation, e.g. of deuterium and helium, can also easily be realised using a plasma source without the need for a complicated dual ion beam set-up. On the other hand, there is usually a broad spectrum of ions and reactive neutrals emanating from a deuterium low-temperature plasma. In contrast to an ion beam, the particle species from a plasma source cannot be separated. Furthermore, impurity ions, which can be easily suppressed in ion beams, may be present in the plasma flow. This makes the quantification of these species much more complex than for an ion beam experiment. Despite this complexity, the exposure of samples to a low-temperature plasma resembles more closely the conditions in the divertor region of a fusion experiment [2] and is therefore desirable.

An existing plasma source with a biased sample holder ('PlaQ') was thoroughly characterised. A set of suitable operating parameters for deuterium operation was determined, with focus on good long-term stability and reproducibility. For these parameters, the absolute flux as well as the mass and energy distribution of the impinging ions was then quantified. In addition an attempt to determine the flux of atomic deuterium is presented. 


\section{Experimental}

\subsection{Plasma source}

The plasma source PlaQ (see Fig. 1) is a remote electron-cyclotron-resonance (ECR) discharge with a biased sample holder. The magnetic field necessary for the electron cyclotron resonance is created by a single magnetic coil. The microwaves from a magnetron with $\mathrm{P}_{\max }=1200 \mathrm{~W}$ and $f=2.45 \mathrm{GHz}$ are coupled into the vacuum vessel from the high $B$ field side by a waveguide terminated by a quartz window. The plasma is confined to a cylindric steel mesh cage with a height of $15 \mathrm{~cm}$ and a diameter of $14 \mathrm{~cm}$. The bottom of the cage is made of a solid steel plate with a circular aperture (diameter: $5 \mathrm{~cm}$ ). Through this aperture a freely expanding plasma beam reaches a sample holder. A wide mesh tungsten grid in the aperture (grid spacing approximately $1 \mathrm{~cm}$ ) shields the samples from the microwave radiation. The distance between the aperture and the sample holder is $10 \mathrm{~cm}$. The whole sample holder (diameter: $7.0 \mathrm{~cm}$ ) is homogeneously irradiated. The lateral homogenity of the plasma beam was tested by eroding amorphous hydrogenated carbon thin films.

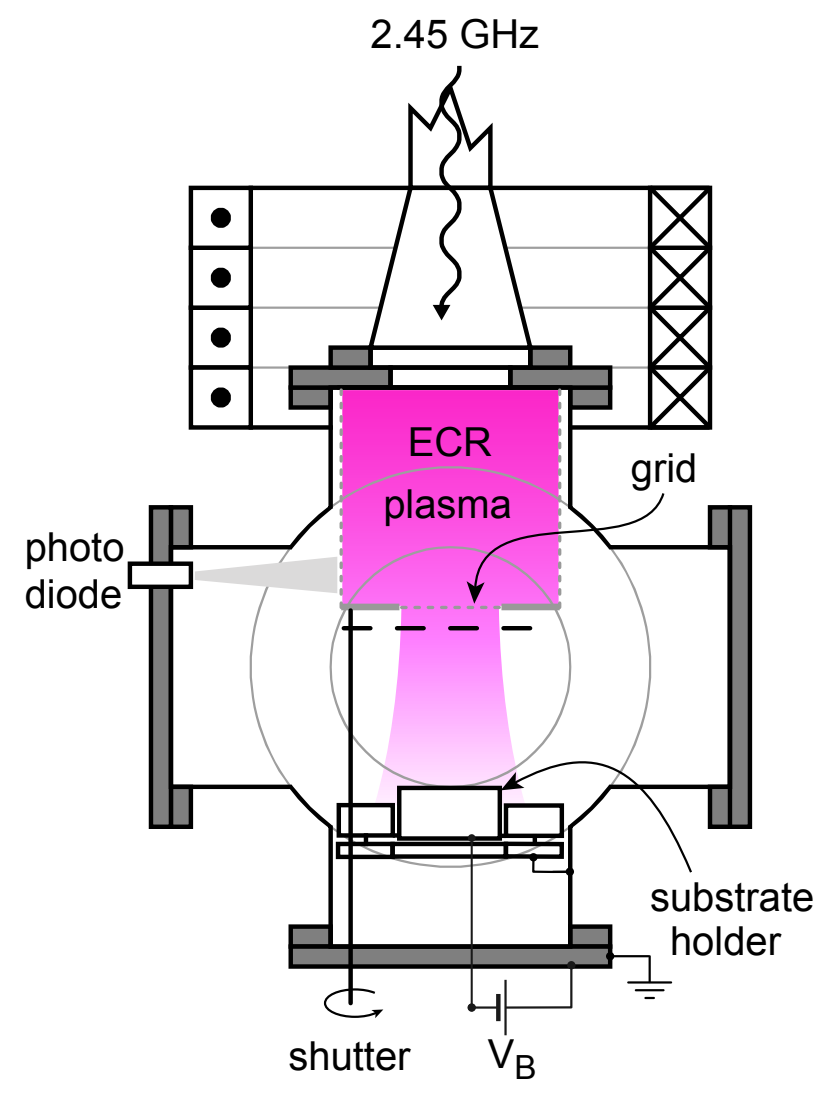

Figure 1. Schematic representation of the PlaQ plasma source.

To assure good thermal contact, samples are clamped to the substrate holder by screws. Because of that, no load lock chamber or sample transfer is installed, but the whole plasma chamber is vented with nitrogen in order to insert or remove samples. 
Typically, a base vacuum of better than $10^{-4} \mathrm{~Pa}$ can be reached within 4 hours after a sample change. The base vacuum is in the order of 2 to $3 \cdot \mathbf{1 0}^{-\mathbf{5}} \mathrm{Pa}$ and is typically reached after approximately 12 hours of pumping.

The plasma beam can be blocked with a shutter underneath the ECR cage aperture to allow for a burn-in time of the plasma without exposing the sample to the beam. This is necessary because the ECR cage heats up to approximately $400 \mathrm{~K}$ due to interaction with the plasma over a time of approximately 30 minutes. During this time, the surface of the cage is cleaned of adsorbed impurities as measurements with a residual gas analyser have shown (see section 2.2). The burn-in phase is accompanied by an increase in the total light emission from the plasma (see section 2.2). Once the light emission has reached a saturation value, the plasma conditions are stable on the timescale of an implantation run (typically between 1 hour and 3 days).

The sample holder is electrically isolated from the vacuum chamber and can be biased up to $-600 \mathrm{~V}$ by a DC power supply for electrically conductive samples (i.e. tungsten). For electrically insulating samples or samples with an insulating coating, DC biasing cannot be used because this causes arcing. In this case, the bias is produced by a supplementary asymmetric capacitive RF discharge. However, here we only present the ion fluxes for DC biasing.

For temperatures between room temperature and $450 \mathrm{~K}$, an open circuit thermostat with silicon oil is used to stabilize the sample holder temperature. In this case, the sample holder and its support structure are made of copper and a boron nitride insulator is used in order to maximize the heat conductivity between the sample and the heat sink. For higher temperatures up to $1000 \mathrm{~K}$, the sample holder is radiation heated by a BORALECTRIC ${ }^{\mathbb{R}}$ heater element $\ddagger$. In this set-up the copper parts are replaced by stainless steel and a glass ceramic insulator is used to minimize heat losses by thermal conductivity.

\subsection{Standard diagnostics}

During the implantation procedure, a number of operating parameters of the plasma source are monitored, namely the sample holder temperature, the total plasma light emission, the magnetic field coil current, the forward and reflected microwave power and the gas pressure, the sample holder bias and in DC mode also the resulting electrical current.

The sample holder temperature is measured independently by a thermocouple that is pressed against the sample holder from below and by a microbolometer infrared camera that directly measures the temperature of the plasma-facing surface. The total plasma light emission is measured by a broadband silicon photo diode whose line of sight goes through the ECR cage from the side as depicted in Fig. 1. It serves as an indicator for the reproducibility and stability of the ECR discharge. As will be shown below, the stability and reproducibility of the ion flux can be qualitatively monitored during DC operation by measuring the electrical current to the sample holder. The bias voltage during RF biasing is measured by using a low pass filter that only allows the DC component to pass. Microwave power, coil current and gas pressure are recorded for maintenance purposes.

$\ddagger$ Distributed by tectra GmbH, Reuterweg 65, D-60323 Frankfurt/Main 
For monitoring the impurity concentration in the vacuum chamber, a residual gas analyser with a quadrupole mass spectrometer is connected to the plasma chamber. It is installed in a differentially pumped chamber $1.5 \mathrm{~m}$ away from the plasma chamber to minimize disturbance from the magnetic field. Because of that long tube with $35 \mathrm{~mm}$ inner diameter it can only measure stable neutrals. During plasma operation (plasma pressure typically larger than $10^{-1} \mathrm{~Pa}$ ) neutral species can be sampled through an orifice $50 \mu \mathrm{m}$ in diameter to meet the low pressure $\left(<10^{-4} \mathrm{~Pa}\right)$ requirements neccesary for the residual gas analyser. This system is used, for example, to monitor the pumpdown procedure after installing new samples, to trace impurity release during the burn-in time of the plasma, or to monitor volatile erosion products. Fig. 2 shows an example for a measurement of impurity levels during a typical pumpdown and plasma operation sequence.

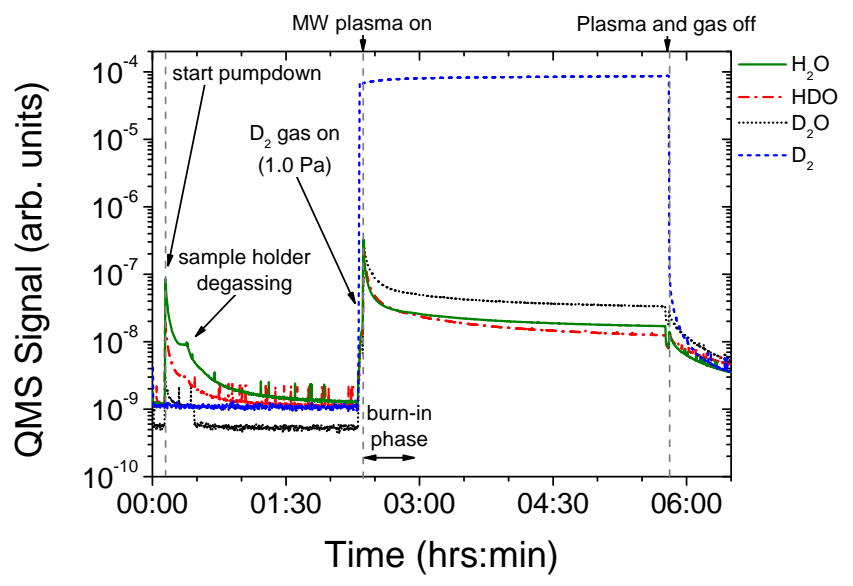

Figure 2. Quadrupole mass spectrometer measurement during pumpdown and plasma operation. The sample holder was heated to approximately $400 \mathrm{~K}$ during pumpdown for degassing. The main impurities are $\mathrm{H}_{2} \mathrm{O}, \mathrm{HDO}$ and $\mathrm{D}_{2} \mathrm{O}$. For comparison the signal of the operating gas $\mathrm{D}_{2}$ is also shown. The individual signals are corrected for relative sensitivity.

The monitoring of these various operating parameters has proven that the implantation process is stable over extended periods of more than 60 hours and can be accurately reproduced from experiment to experiment.

\subsection{Retarding field analyzer}

For the absolute quantification of the mass-integrated ion flux to the sample holder and the energy distribution of the ion flux, a retarding field analyzer (RFA) was used (Fig. 3). It was installed in the vacuum chamber instead of the sample holder and consists of a mock-up sample holder with a $100 \mu \mathrm{m}$ aperture to which the bias voltage $V_{R}$ is applied. Behind the aperture three gold grids with known transparency and a cup-shaped collector electrode are mounted. The whole electrode system is differentially pumped in order to minimise collisions of the ions with residual gas molecules. The first grid ('repeller') is biased with the repeller voltage $V_{R}=-54 \mathrm{~V}$ relative to the sample plate mock-up in order to repel electrons from the plasma. The following two grids serve as an energy 
discriminator for the ions and are set to a variable positive potential $V_{D}$ with respect to ground potential. The collector electrode is set to the same potential as the discriminator grids in order to minimise the amount of secondary electrons escaping from the collector. The current $I_{C}$ to the collector electrode is measured as a function of $V_{D}$ by a Keithley Model 6517A electrometer.

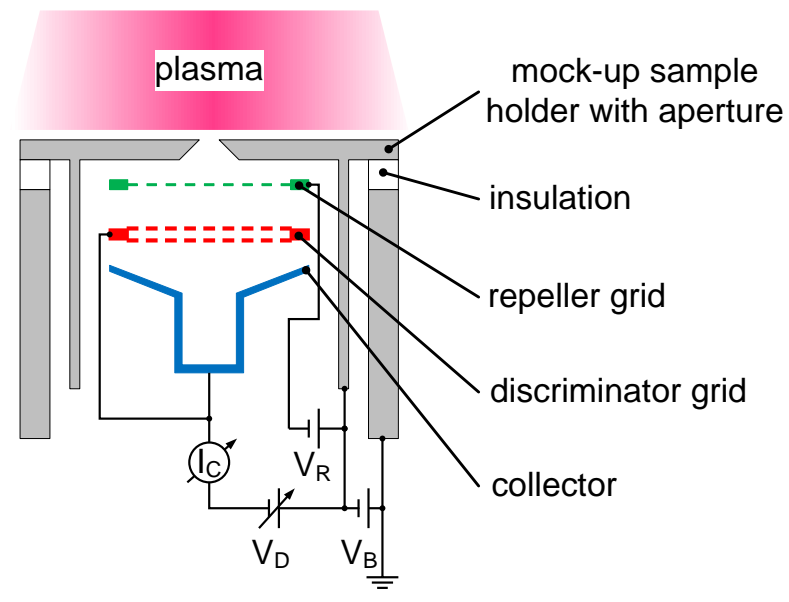

Figure 3. Schematic representation of the retarding field analyser (RFA).

For singly charged ions (i.e. all positive hydrogen ion species), the collector current $I_{C}\left(V_{D}\right)$ is given by the integral function

$$
I_{C}\left(V_{D}\right)=\int_{e \cdot V_{D}}^{\infty} d E A \cdot e \cdot \gamma_{E}(E)
$$

with $\gamma_{E}(E)$ being the differential flux density of ions per energy interval and $A$ the area of the $100 \mu \mathrm{m}$ aperture. The derivative $d I_{C} / d V_{D}$ is therefore proportional to $\gamma_{E}(E)$. An example is presented in Fig. 4. It should be noted that $\gamma_{E}(E)$ is not equivalent to the ion energy distribution $f_{E}(E)=d n_{i}(E) / d E$ ( $n_{i}$ being the ion density), but closely related to the ion velocity distribution $f_{v}(v)=d n_{i}(v) / d v$ [6]. In fact, $\gamma_{E}(E)$ is essentially a rescaling of the quantity $v \cdot f_{v}(v)$ to units of energy instead of velocity. However, $f_{v}(v)$ is not the most intuitive quantity to describe an implantation experiment. The differential ion flux density $\gamma_{E}(E)$ is better suited to this purpose because it directly reflects the flux of ions impinging on the sample with a certain kinetic energy.

During RFA measurements, the current to the mock-up sample holder was also measured. This current turned out to depend linearly on the ion flux for a fixed sample holder bias voltage. It can, therefore, serve as an easily measurable quantity that allows to monitor the ion flux during implantations. This is discussed in more detail in section 3.4.

\subsection{Plasma monitor}

The mass distribution of the ions impinging on the sample holder was measured with a plasma monitor (Model HIDEN EQP 300). Similar to the RFA set-up a mock-up sample holder was installed instead of the real sample holder. In order to shield the plasma 

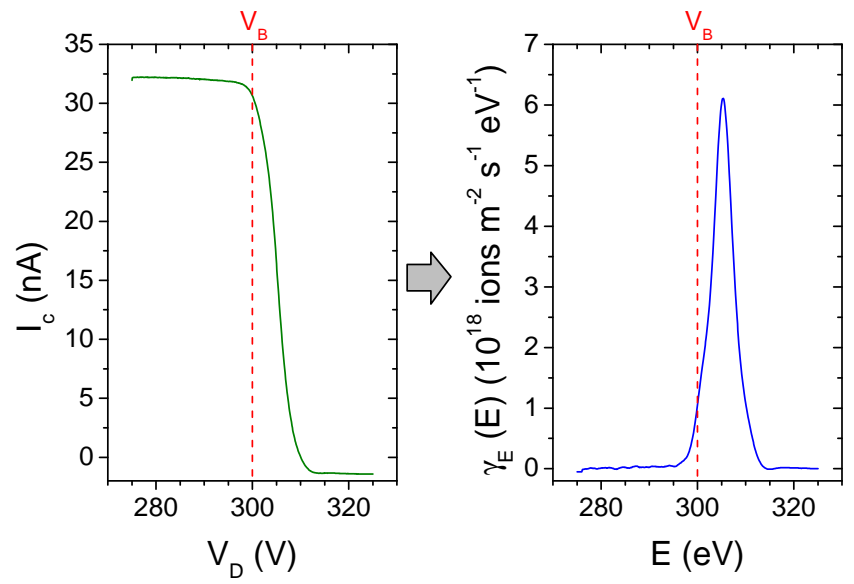

Figure 4. Collector current $I_{C}$ versus discriminator voltage $V_{D}$ (left graph) and differential ion flux density $\gamma_{E}$ versus ion energy (right graph) for a DC bias voltage of $-300 \mathrm{~V}$. The $\mathrm{D}_{2}$ gas pressure was $1.0 \mathrm{~Pa}$, the nominal microwave input power was $144 \mathrm{~W}$.

monitor from the magnetic field necessary for the ECR discharge, the mock-up sample holder was constructed from soft iron. An additional soft iron shielding cylinder was placed around the ion optics of the plasma monitor. The plasma monitor is differentially pumped in order to maintain a pressure of about $10^{-7}$ to $10^{-6} \mathrm{~Pa}$ during operation. The aperture in the mock-up sample holder is only $10 \mu \mathrm{m}$ in contrast to the $100 \mu \mathrm{m}$ aperture that was used in the RFA set-up. The measurement system of the HIDEN EQP consists of ion extraction and focusing optics, a $45^{\circ}$ electrostatic energy analyser and a quadrupole mass spectrometer with a channeltron detector operating in single ion counting mode.

Because the absolute flux of ions and its energy distribution are more reliably measured with the RFA, the plasma monitor signal is integrated over the whole ion energy range to derive only the relative abundance of each ion species. This is permissible since the signals for $\mathrm{D}^{+}, \mathrm{D}_{2}^{+}$and $\mathrm{D}_{3}^{+}$all have the same qualitative dependence on the retardation potential of the energy analyser. The integrated values are weighted by the mass dependent transmission of the plasma monitor. The transmission factors were experimentally determined by [7] and are proportional to the empirical function $\exp \left(-\frac{m}{18.28}\right)$ with $m$ being the ion mass in atomic mass units. The results are then scaled to the total ion flux measured with the RFA in order to obtain the mass-resolved ion flux. Multiplying the fluxes of the individual ions with the number of deuterons per ion yields the flux of deuterons per parent ion, from which the total deuteron fluence accumulated during the implantation can be calculated. It has to be kept in mind that the different parent ions deliver deuterons with energies of $e \cdot V_{B}, e \cdot V_{B} / 2$ and $e \cdot V_{B} / 3$ for $\mathrm{D}^{+}, \mathrm{D}_{2}^{+}$and $\mathrm{D}_{3}^{+}$respectively. 


\section{Results}

\subsection{Power variation}

The microwave input power for the ECR plasma was varied between 60 and $180 \mathrm{~W}$ at a constant $\mathrm{D}_{2}$ gas pressure of $1.0 \mathrm{~Pa}$. For powers lower than $60 \mathrm{~W}$ the plasma could not be maintained, and for powers significantly higher than $180 \mathrm{~W}$ the temperature of the ECR cage and the vacuum vessel near the cage exceeded safety limits after approximately 30 minutes. The power variation series were measured with the sample holder at floating potential and with an applied DC sample holder bias of $V_{B}=-200 \mathrm{~V}$.

Fig. 5 shows the combined result of the RFA and plasma monitor measurements for the power variation at floating potential and a constant pressure of $1 \mathrm{~Pa}$. The total ion flux increases for powers up to $130 \mathrm{~W}$, while for powers between $130 \mathrm{~W}$ and $180 \mathrm{~W}$ the ion flux stays in good approximation constant. The relative contributions from the different ion species do not significantly depend on the microwave power. The dominant ion for these conditions is always $D_{3}^{+}$. For $V_{B}=-200 \mathrm{~V}$ (not shown here), the dependence of the ion flux on the microwave power shows the same saturation effect for $130 \mathrm{~W}$ and higher, although the absolute fluxes are systematically higher by approximately a factor of 1.8. At 170 and $180 \mathrm{~W}$ microwave power, the discharge became unstable for the biased sample holder which resulted in strong fluctuations of the ion flux. The influence of the sample holder bias on the ion flux is discussed in detail in section 3.3.

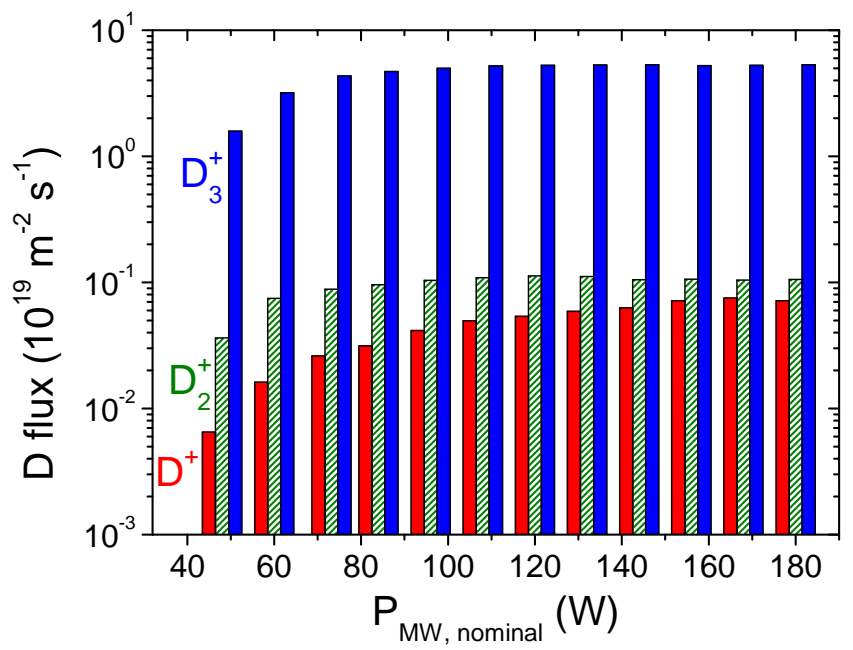

Figure 5. Ion-species-resolved total deuteron flux as a function of the microwave input power, measured at constant $\mathrm{D}_{2}$ gas pressure of $1.0 \mathrm{~Pa}$ and with the sample holder at floating potential. In the figure the number of deuterons carried by a molecular ion is taken into account, i.e. a $\mathrm{D}_{2}^{+}$ion contributes 2 and a $\mathrm{D}_{3}^{+}$ion 3 deuterons to the total flux.

The differential ion flux distribution $\gamma_{E}$ depends strongly on the microwave input power and exhibits bi- and even trimodal shapes for powers below $130 \mathrm{~W}$. Above this value however, $\gamma_{E}$ is independent from the microwave power and approximately monomodal. This is also the case for a biased sample holder.

Based on these results, $144 \mathrm{~W}$ was chosen as the operating point for all future 
measurements and implantations in order to make the discharges and the ion flux robust against small fluctuations of the microwave power.

\subsection{Pressure variation}

The $\mathrm{D}_{2}$ gas pressure was varied between 0.3 and $6.0 \mathrm{~Pa}$ at a constant microwave power of $144 \mathrm{~W}$. As for the microwave power variation in section 3.1, two series were measured, one with the sample holder at floating potential and one with a DC bias of $V_{B}=-200 \mathrm{~V}$.

Fig. 6 shows the the combined result of the RFA and plasma monitor measurements as a function of the gas pressure for a floating sample holder. The total deuteron flux decreases by nearly two orders of magnitude from $1.2 \cdot 10^{20} \mathrm{D} \mathrm{m}^{-2} \mathrm{~s}^{-1}$ down to $1.7 \cdot 10^{18} \mathrm{D} \mathrm{m}^{-2} \mathrm{~s}^{-1}$ if the pressure is increased from 0.3 to $6.0 \mathrm{~Pa}$. The gas pressure also influences the ion species composition. In the whole investigated pressure range the deuteron flux carried by $\mathrm{D}_{3}^{+}$ions dominates the total deuteron flux, and $\mathrm{D}_{3}^{+}$ions are even the most abundant ion species. At lower pressures, however, the fraction of deuterons from $\mathrm{D}_{2}^{+}$ions increases considerably, while at $6.0 \mathrm{~Pa}$ the $\mathrm{D}_{2}^{+}$contribution is hardly above the detection limit. The observed abundances of the different deuterium ion species can be rationalised by taking into account that only $\mathrm{D}^{+}$and $\mathrm{D}_{2}^{+}$can be produced by direct ionisation. The effective rate coefficient for the production of $D_{2}^{+}$from $D_{2}$ is considerably higher than for the production of $\mathrm{D}^{+}[8]$. The $\mathrm{D}_{3}^{+}$ion can only be produced by the ion-molecule reaction

$$
\mathrm{D}_{2}^{+}+\mathrm{D}_{2} \longrightarrow \mathrm{D}_{3}^{+}+\mathrm{D}
$$

This reaction has a very high cross section for the near-thermal ion energies that are typical for low-temperature plasmas. Since this is a reaction with the neutral $D_{2}$ gas, it becomes more efficient for higher pressures. Ion-molecule reactions of $\mathrm{D}^{+}$and $\mathrm{D}_{3}^{+}$ with $\mathrm{D}_{2}$ become relevant only at ion energies of more than $10 \mathrm{eV}$ [9] and can thus be neglected for our conditions. Therefore, the gas pressure influences above all the balance between $\mathrm{D}_{2}^{+}$and $\mathrm{D}_{3}^{+}$ions by the conversion reaction of $\mathrm{D}_{2}^{+}$into $\mathrm{D}_{3}^{+}$. This means that

for higher $\mathrm{D}_{2}$ gas pressure the $\mathrm{D}_{2}^{+}$concentration in the plasma is increasingly reduced while $\mathrm{D}_{3}^{+}$ions are produced.

The differential ion flux distributions $\gamma_{E}$ as measured by the RFA become significantly narrower for higher pressures and remain monomodal. For a sample holder bias of $V_{B}=-200 \mathrm{~V}$ the ion flux and the contributions by the different deuterium ion species show the same qualitative behaviour as with the sample holder at floating potential. The absolute values are systematically higher by a factor between 1.5 and 2, similar to the results for the power variation (section 3.1).

Taking all that into account, a pressure of $1.0 \mathrm{~Pa}$ is a good compromise for deuterium implantations. On the one hand, the total deuteron flux is high enough to allow the accumulation of large fluences within a reasonable implantation time. On the other hand the ion flux is clearly dominated by a single ion species (i.e. $\mathrm{D}_{3}^{+}$) and has a reasonably narrow energy distribution.

The strong dependence of the ion flux on the pressure by nearly two orders of magnitude also allows to vary the deuteron flux to the sample in order to investigate flux-dependent processes or to reduce the heat load delivered by the ions if it exceeds the 


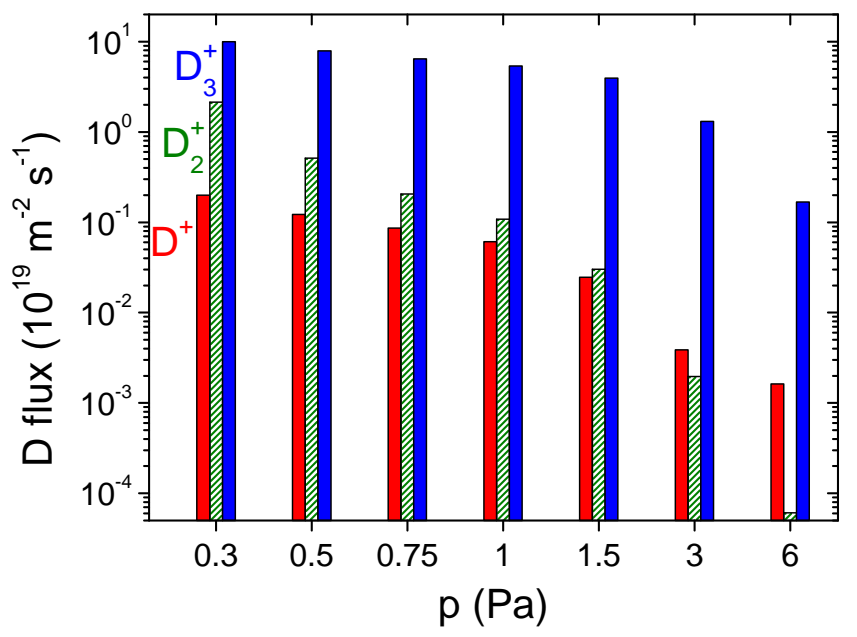

Figure 6. Ion-species-resolved deuteron flux as a function of the $\mathrm{D}_{2}$ gas pressure, measured at a constant microwave input power of $144 \mathrm{~W}$ and with the sample holder at floating potential.

cooling capacity of the sample holder. However, it has to be kept in mind that varying the pressure changes the ion species composition as well as the plasma potential, which affects the energy per deuteron for a given bias potential.

\subsection{Bias variation}

Fig. 7 shows the differential ion flux distributions $\gamma_{E}$ measured with the RFA for seven different bias potentials applied to the mock-up sample holder. The measurements were all performed at $144 \mathrm{~W}$ microwave input power and $1.0 \mathrm{~Pa} \mathrm{D}_{2}$ pressure. The shape of the of the ion flux distribution is nearly conserved for sample holder biases ranging from floating potential up to $V_{B}=-600 \mathrm{~V}$ DC bias. The total deuteron flux increases with increasing bias from $5.6 \cdot 10^{19}$ to $1.1 \cdot 10^{20} \mathrm{D} \mathrm{m}^{-2} \mathrm{~s}^{-1}$. The largest part of this variation takes place between floating potential and a $D C$ bias of $V_{B}=-100 \mathrm{~V}$. For $V_{B} \geq-300 \mathrm{~V}$ the total deuteron flux saturates although the peak value of $\gamma_{E}$ decreases slightly. This is accounted for by a slight broadening of the ion flux distribution. For floating potential, the FWHM is $3.9 \mathrm{eV}$ while for $-600 \mathrm{~V}$ it is $6.6 \mathrm{eV}$. It should be noted however that the peak shape is not Gaussian, but slightly asymmetric and has shoulders at higher and lower energies that lead to a width at the base of the distribution between 15 and $20 \mathrm{eV}$. The ion species composition determined by plasma monitor measurements was approximately the same for all values of $V_{B}$. This shows that there are no or only very few collisions in the sheath region where the ions are accelerated. The lowest ion energy achievable for the chosen parameter set is $15 \mathrm{eV}$ or $5 \mathrm{eV}$ per deuteron, respectively. In addition, one can see in Fig. 7 that the maximum of the ion flux distribution is always at a slightly higher energy than the applied bias value. This difference is due to the plasma potential defined by the chosen pressure and microwave power. The fact that this difference is nearly independent from the applied bias voltage proves the successful decoupling of the ion energy from the plasma generation. 


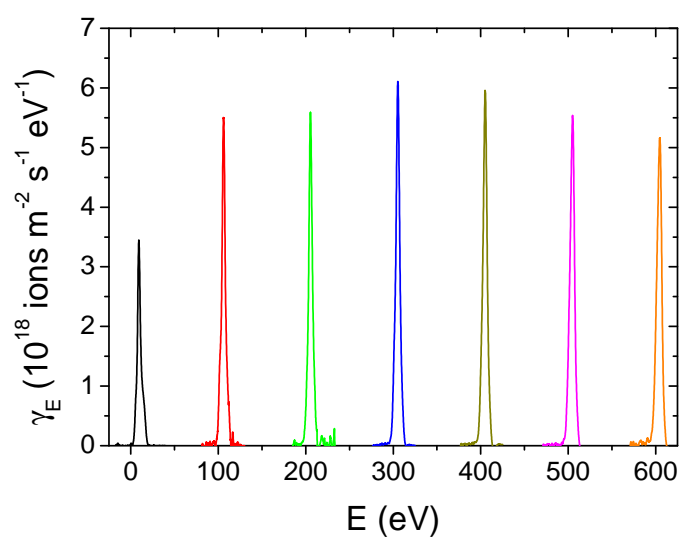

Figure 7. Differential ion flux distribution $\gamma_{E}$ for the sample holder at floating potential and biased at $-100 \mathrm{~V},-200 \mathrm{~V},-300 \mathrm{~V},-400 \mathrm{~V},-500 \mathrm{~V}$ and $-600 \mathrm{~V}$. The measurements were performed at $144 \mathrm{~W}$ microwave input power and $1.0 \mathrm{~Pa}_{2}$ gas pressure.

\subsection{Sample holder current}

For any DC bias other than floating potential, the current to the sample holder can be measured. The ion flux density corresponding to this current can then be compared to the ion flux density measured by the RFA. It turned out that the current measurement delivers identical flux density values as measured with the RFA for a sample bias just above floating potential, but systematically larger values with increasing bias. This increasing deviation can be explained by secondary electrons emitted from the sample holder surface due to the impact of energetic ions. An emission of a negative charge carrier cannot be distinguished from the impact of an additional positively charged particle. Increasing secondary electron emission, therefore, results in an apparent increase of the sample holder current. At constant bias, however, the sample holder currend turned out to be in good approximation proportional to the ion flux during variations of the $\mathrm{D}_{2}$ gas pressure and the microwave power. This is illustrated in Fig. 8 for a pressure variation at $-200 \mathrm{~V}$ bias. The value from the sample holder current measurement is proportional to the RFA value, but systematically 1.5 times higher due to secondary electron emission. Because of this proportionality the sample holder current can be used for real-time monitoring of the total ion flux during deuterium implantations.

\subsection{Neutral deuterium atom flux}

Although the atoms are in contrast to the ions not accelerated in the sheath their chemical reactivity is high. In addition to the fact that they alone may cause reactions at the surface it is well known that synergistic effects can take place if atomic and ionic species interact with surfaces simultaneously $[10,11,12,13,14]$. Especially at low ion energies the so-called chemical sputtering [14] sets in where the erosion yield can be much larger than the yield expected for the neutral and ion species alone. This was clearly demonstrated for the erosion of hydrocarbon films $[10,11,12,13]$. In the case 


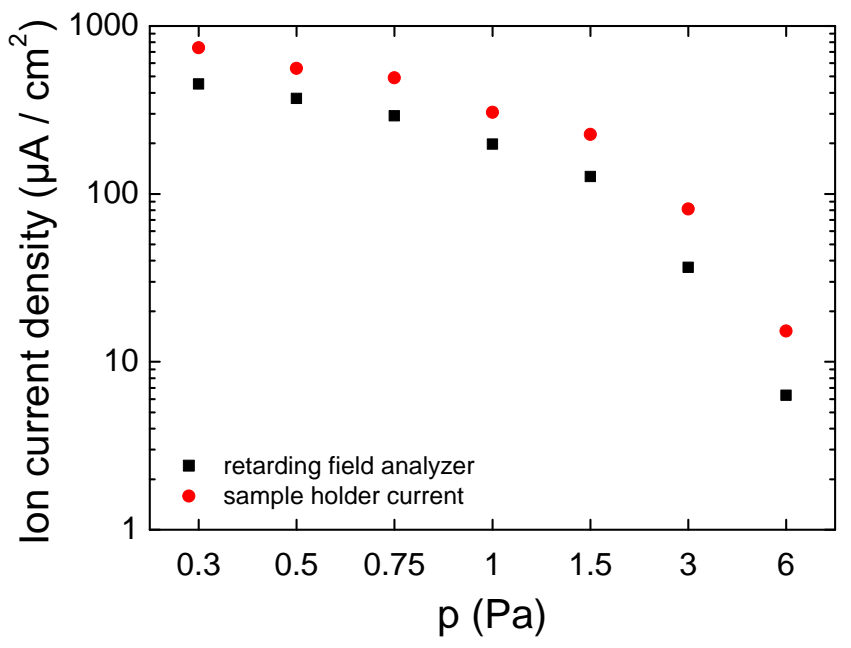

Figure 8. Total ion current density derived from retarding field analyzer and sample holder current measurements for a DC bias of $-200 \mathrm{~V}$. The measurements were taken at a constant microwave input power of $144 \mathrm{~W}$.

of metals such as $\mathrm{W}$ chemical sputtering can be excluded, but for retention studies in particular at low bias voltages deuterium atoms might considerably contribute to the observed effects. It is, therefore, important to assess the fluxes of atomic deuterium.

In fact very different dissociation degrees were reported in literature. For a GEC cell with steel walls Chérigier et al. [15] reported dissociation degrees in the range of 1 to $5 \%$ for capacitively coupled RF plasmas $(13.56 \mathrm{MHz})$ in pure hydrogen (pressure range 25 to $85 \mathrm{~Pa}$ ). Jolly and Booth found for a capacitively coupled RF discharge in hydrogen at $133 \mathrm{~Pa}$ a dissociation degree of 0.005 . For microwave discharges in hydrogen/helium mixtures at $12 \mathrm{~Pa}$ density ratios of $\mathrm{H} / \mathrm{H}_{2}$ between approximately 0.01 and 0.2 for varying $\mathrm{H}_{2}$ concentrations in He between 3 and $90 \%$ are reported in [16]. This illustrates that the dissociation degree and with it the absolute hydrogen flux reaching a surface can vary largely for different discharges.

But compared with the ion flux, the flux of atomic deuterium is difficult to measure. One approach is optical emission spectroscopy [17], but requires significant effort for an accurate intensity calibration of the spectrometer as well as an optical system with a good spatial resolution. Other quantitative optical methods like TALIF $[18,15,19]$ are far too elaborate to be applied here. An attempt to measure the flux of atomic hydrogen with ionization threshold mass spectrometry with the plasma monitor failed because of the huge background of molecular hydrogen which raises the detection limit.

We use here an alternative method initially suggested to quantify thermal atomic deuterium beams [20]. The method is based on the erosion of a plasma-deposited amorphous hydrogenated carbon thin film $(\mathrm{a}-\mathrm{C}: \mathrm{H})$. From the known erosion yield and the measured erosion rate the absolute particle flux can be determined because the density of the eroded film is known [21]. But application of that method in a plasma environment is not straightforward, because due to synergistic interactions the erosion rates of the individual species fluxes cannot simply be added $[10,11]$. However, with the absolutely determined ion fluxes from the retarding field analyser and the ion flux 
composition determined with the plasma monitor the atomic flux can be estimated. This is done by assuming reasonable yields for the ion species, calculating therewith expected rates based on the measured ion flux and comparing the result with the measured rates. Any erosion exceeding the expected value can then be attributed to the deuterium atom flux.

The result of such measurements is shown in Fig. 9. Dense a-C:H films with a $\mathrm{H}$ content of about $30 \%$ [21] were plasma deposited in-situ on single-crystalline silicon substrates by a methane capacitively coupled RF discharge $(13.56 \mathrm{MHz})$ applying a self-bias voltage of $-200 \mathrm{~V}$. In the following the films were eroded by a deuterium ECR plasma. During erosion the substrate was kept at floating potential to minimize sputtering. The microwave input power was again $144 \mathrm{~W}$. Growth and erosion of the film was monitored in real time by in-situ ellipsometry [22]. The measured rate is shown in Fig. 9 in logarithmic scale as a function of plasma pressure for two sample temperatures. For both temperatures, the rate drops by about a factor of 20-40 if the pressure is increased from 0.3 to $6.0 \mathrm{~Pa}$. The same qualitative dependence was observed for the total ion flux as shown in Fig. 6.

In addition to these measured rates, erosion rates were also calculated based on the measured ion flux for three different assumptions about the erosion yields. The first pessimistic assumption is that the erosion yield of a low energy $D_{3}^{+}$ion is as small as the yield of thermal atomic $\mathrm{D}$, which is $3 \cdot 10^{-4}$ at $310 \mathrm{~K}$ and $2 \cdot 10^{-2}$ at $650 \mathrm{~K}$ [13]. Assuming a yield of $3 \cdot 10^{-4}$ the expected erosion rate measured at $310 \mathrm{~K}$ is more than three orders of magnitude smaller than the measured one. One would therefore conclude, that the flux of atomic deuterium is three orders of magnitude larger than the ion flux. The more realistic, second assumption is that the erosion yield at room temperature is $4 \cdot 10^{-2}$ as determined by beam experiments with $\mathrm{D}_{3}^{+}$ions [23]. With this yield the expected erosion rate measured at $310 \mathrm{~K}$ is still one order of magnitude smaller than the measured one as it can be seen in Fig. 9. If we further assume that the erosion yield of the impinging deuterium atoms remains unaffected by the simultaneously impinging ion flux at $3 \cdot 10^{-4}$ one would infer a flux of atomic deuterium of $4 \cdot 10^{-2} / 3 \cdot 10^{-4} \times 10 \approx 1000$ times larger than the ion flux. On the other hand, if we assume that the yield for the atoms is increased due to the simultaneous ion flux to the same yield as that of the ions then we would arrive at a flux of atomic deuterium which is about one order of magnitude larger than the flux of ions. The second calculated curve in Fig. 9 assumes an erosion yield for the ions of 0.1 at $650 \mathrm{~K}$ as known from ion beam experiments [23]. With this yield the calculated rate is again about one order of magnitude smaller than the measured rate at $650 \mathrm{~K}$. To estimate the flux of atomic deuterium from this excess erosion we again have to assume an erosion yield for the deuterium atoms. If we make the same assumption as above that the atoms have a yield identical to the yield of the ions, i.e. 0.1, we arrive at a deuterium atom flux which is 10 times higher than the ion flux. But if we assume that the yield for the atoms remains unchanged at $2 \cdot 10^{-2}$ the estimated atom flux would be an additional factor of 50 higher.

We are convinced that assuming for the atoms the identical yield as for the ions is the more realistic choice. This assumption has also be made in the past for ion beam experiments at higher ion energies $\left(E_{i o n} \geq 100 \mathrm{eV}\right)[24,25]$ and results independently from the extrapolation of the model devised by Hopf et al. $[10,11,26]$. Basically 
this assumption means that in the presence of ion bombardment the reactivity of the surface towards reactions with impinging low energy atomic hydrogen atoms is enhanced compared with a surface that is not bombarded. This is the fundamental explanation of the observed synergistic enhancement for chemical sputtering of carbon materials in the presence of atomic hydrogen. In conclusion the measurements show that the flux of atomic deuterium is at least one order of magnitude larger than the deuteron flux delivered by the ions. This conclusion is also supported by an estimation of the molecular flux of atomic deuterium to the sample holder surface which is given by

$$
\Gamma_{\mathrm{D}}=n_{\mathrm{D}} \cdot v_{\text {thermal }} / 4 \text {. }
$$

Assuming that the density ratio of atomic $D$ to molecular $D_{2}$ is in the order of 0.01 at the typical operating pressure of $1.0 \mathrm{~Pa}$, and that the gas temperature is approxiately $400 \mathrm{~K}, \Gamma_{\mathrm{D}}$ is of the order of $10^{21} \mathrm{~m}^{-2} \mathrm{~s}^{-1}$. This is in good agreement with the results of the erosion method presented here.

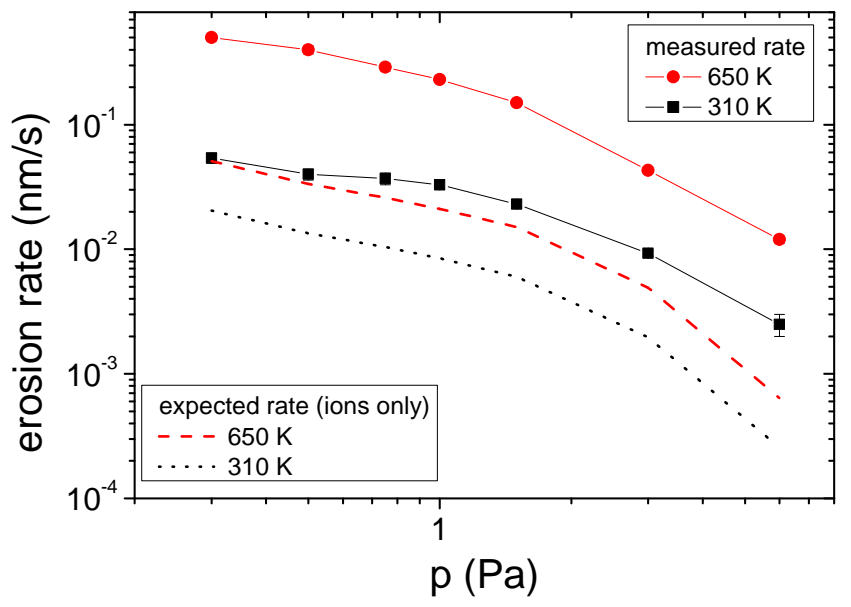

Figure 9. Measured (symbols) and calculated (dashed lines) erosion rates of dense a-C:H films exposed to deuterium plasmas at a microwave input power of $144 \mathrm{~W}$ and the sample holder at floating potential as a function of $\mathrm{D}_{2}$ gas pressure. The calculated rates are based on the measured ion flux only and assuming yields of $0.04(310 \mathrm{~K})$ and $0.1(650 \mathrm{~K})$.

\section{Conclusions}

The ion flux from the remote ECR plasma source PlaQ was thoroughly quantified for plasma conditions optimised for the implantation of deuterium. A set of operating parameters was found where the source is able to deliver a constant flux of deuterium ions over long timespans. The ion flux is stable against small fluctuations of the microwave power at the typical operating point of $144 \mathrm{~W}$. It can be varied over approximately two orders of magnitude by varying the operating pressure between 0.3 and $6.0 \mathrm{~Pa}$. The reproducibility and stability of the ion flux during implantations can be monitored by measuring the current to the sample holder.

For operating pressures of $0.5 \mathrm{~Pa}$ or larger over $90 \%$ of the incident deuterons are carried by $\mathrm{D}_{3}^{+}$ions, while the rest is carried by $\mathrm{D}_{2}^{+}$and $\mathrm{D}^{+}$. The energy distribution of 
the incident ion flux is narrow and peaked. Its shape does not significantly change when a DC bias is applied to the sample holder, only the center of weight is shifted according to the bias. It has to be kept in mind that although $\mathrm{D}_{3}^{+}$is the dominant species, the incident ion flux is not mass filtered and that the minority ion species $\mathrm{D}_{2}^{+}$and $\mathrm{D}^{+}$have twice respectively thrice as much energy per deuteron.

The flux of atomic deuterium was estimated by erosion of plasma deposited, dense $\mathrm{a}-\mathrm{C}: \mathrm{H}$ films. The flux of atomic deuterium is at least one order of magnitude larger than the deuteron flux delivered by the ions.

For the defined standard operating parameters, i.e., a $\mathrm{D}_{2}$ plasma at $p=1.0 \mathrm{~Pa}$, $P_{M W}=144 \mathrm{~W}$ and the sample holder at floating potential, the total deuteron flux in form of ions is $5.6 \cdot 10^{19} \mathrm{D} \mathrm{m}^{-2}$. $94 \%$ of the deuterons are carried by $\mathrm{D}_{3}^{+}, 3 \%$ by $\mathrm{D}_{2}^{+}$ and $3 \%$ by $\mathrm{D}^{+}$ions. The flux increases monotonically by about a factor of 2 if the bias voltage is increased to $-600 \mathrm{~V}$. With the absolute quantification of the ion flux and ion species distribution it is now possible to perform reliable and well defined deuterium implantation experiments.

[1] W. Jacob. Redeposition of hydrocarbon layers in fusion devices. J. Nucl. Mater., 337-339:839-846, 2005.

[2] J. Roth, E. Tsitrone, A. Loarte, Th. Loarer, G. Counsell, R. Neu, V. Philipps, S. Brezinsek, M. Lehnen, P. Coad, Ch. Grisolia, K. Schmid, K. Krieger, A. Kallenbach, B. Lipschultz, R. Doerner, R. Causey, V. Alimov, W. Shu, O. Ogorodnikova, A. Kirschner, G. Federici, A. Kukushkin, EFDA PWI Task Force, ITER PWI Team, Fusion for Energy, and ITPA SOL/DIV. Recent analysis of key plasma wall interactions issues for ITER. J. Nucl. Mater., 390-391:1-9, 2009.

[3] A. A. Haasz, J. W. Davis, M. Poon, and R. G. Macaulay-Newcombe. Deuterium retention in tungsten for fusion use. J. Nucl. Mater., 263:889-895, 1998.

[4] M. Poon, A. A. Haasz, J. W. Davis, and R. G. Macaulay-Newcombe. Impurity effects and temperature dependence of D retention in single crystal tungsten. J. Nucl. Mater., 313:199-203, 2003.

[5] P. A. Sauter and M. Balden. Deuterium retention in tungsten-doped carbon films. Physica Scripta, T138:014044 (4pp), 2009.

[6] C. Böhm and J. Perrin. Retarding-field analyzer for measurements of ion energy distributions and secondary electron emission coefficients in low-pressure radio frequency discharges. Rev. Sci. Instrum., 64:31, 1993.

[7] P. Pecher. Quantitative Bestimmung der Teilchenflüsse aus Methan-ECR-Plasmen. Phd thesis (in German), Universität Bayreuth, 1997.

[8] K. Sawada and T. Fujimoto. Effective ionization and dissociation rate coefficients of molecular hydrogen in plasma. J. Appl. Phys., 78:2913-2924, 1995.

[9] A. V. Phelps. Cross-sections and swarm coefficients for $\mathrm{H}^{+}, \mathrm{H}_{2}^{+}, \mathrm{H}_{3}^{+}, \mathrm{H}, \mathrm{H}_{2}$, and $\mathrm{H}^{-}$in $\mathrm{H}_{2}$ for energies from $0.1 \mathrm{eV}$ to $10 \mathrm{keV}$. J. Phys. Chem. Ref. Data, 19(3):653-675, 1990.

[10] C. Hopf, A. von Keudell, and W. Jacob. Chemical sputtering of hydrocarbon films by low-energy $\mathrm{Ar}^{+}$ion and $\mathrm{H}$ atom impact. Nucl. Fusion, 42:L27, 2002.

[11] C. Hopf, A. von Keudell, and W. Jacob. Chemical sputtering of hydrocarbon films. J. Appl. Phys., 94:2373, 2003.

[12] W. Jacob, C. Hopf, and M. Schlüter. Chemical sputtering of carbon materials due to combined bombardment by ions and atomic hydrogen. Phys. Scripta, T124:32-36, 2006.

[13] M. Schlüter, C. Hopf, T. Schwarz-Selinger, and W. Jacob. Temperature dependence of the chemical sputtering of amorphous hydrogenated carbon films by hydrogen. J. Nucl. Mater., 376:33-37, 2008.

[14] W. Jacob and J. Roth. Chemical sputtering. In R. Behrisch and W. Eckstein, editors, Sputtering by Particle Bombardment IV, volume 110 of Topics in Applied Physics, chapter Chemical Sputtering, 
pages 329-400. Springer Verlag, Berlin, 2007.

[15] L. Chérigier, U. Czarnetzki, D. Luggenhölscher, V. Schulz-von der Gathen, and Döbele H.F. Absolute atomic hydrogen densities in a radio frequency discharge measured by two-photon laser induced fluorescence imaging. J. Appl. Phys., 85:696-702, 1999.

[16] U. Fantz. Emission spectroscopy of molecular low pressure plasmas. Contrib. Plasma Phys., 44:508515, 2004.

[17] U. Fantz. Atomic and molecular spectroscopy in low temperature plasmas containing hydrogen and deuterium. Technical Report IPP 10/21, Max-Planck-Institut für Plasmaphysik, Garching, 2002.

[18] J. Jolly and J.-P. Booth. Atomic hydrogen densities in capacitively coupled very high-frequency plasmas in $h_{2}$ : Effect of excitation frequency. J. Appl. Phys., 97:103305 (6pp), 2005.

[19] K. Tachibana. VUV to UV laser spectroscopy of atomic species in processing plasmas. Plasma Sources Sci. Technol., 11:A166-A172, 2002.

[20] T. Schwarz-Selinger, A. von Keudell, and W. Jacob. Novel method for absolute quantification of the flux and angular distribution of a radical beam source for atomic hydrogen. J. Vac. Sci. Technol. A, 18:995, 2000.

[21] T. Schwarz-Selinger, A. von Keudell, and W. Jacob. Plasma chemical vapor deposition of hydrocarbon films: The influence of hydrocarbon source gas on the film properties. J. Appl. Phys., 86:3988, 1999.

[22] A. von Keudell and W. Jacob. Growth and erosion of hydrocarbon films, investigated by in situ ellipsometry. J. Appl. Phys., 79:1092, 1996.

[23] M. Balden and J. Roth. New weight-loss measurements of the chemical erosion yields of carbon materials under hydrogen ion bombardment. J. Nucl. Mater., 280:39-44, 2000.

[24] A. A. Haasz, J. W. Davis, O. Auciello, P. Stangeby, E. Vietzke, K. Flaskamp, and V. Philipps. Synergistic methane formation on pyrolytic graphite due to combined $\mathrm{H}^{+}$ion and $\mathrm{H}^{0}$ impact. J. Nucl. Mater., 145-147:412-416, 1987.

[25] J. W. Davis, A. A. Haasz, and P. C. Stangeby. Hydrocarbon formation due to combined $\mathrm{H}^{+}$ion and $\mathrm{H}^{0}$ atom impact on pyrolytic graphite. J. Nucl. Mater., 155-157:234-240, 1988.

[26] M. Schlüter. Synergismen bei der Erosion von amorphen Kohlenwasserstoffschichten mit niederenergetischen Teilchenstrahlen, eine In-Situ-Ellipsometriestudie. Phd thesis (in German), University of Bayreuth, 2007. 